\title{
Presencia de estrés y otros factores de riesgo de enfermedad cardiovascular entre las enfermeras y abogados de una ciudad de Minas Gerais - Brasil
}

\section{EPresence of stress and other risk factors for cardiovascular disease among nurses and lawyers of a city of Minas Gerais - Brazil Presença de estresse e outros fatores de risco para doenças cardiovasculares em enfermeiros e advogados de um município de Minas Gerais - Brasil}

\author{
Aline Daniele Santana Silva ${ }^{1}$, Olga de Fátima Leite Rios ${ }^{2}$, Aline do Carmo França-Botelho ${ }^{3}$ \\ ${ }^{1}$ Graduada em Enfermagem pelo Centro Universitário do Planalto de Araxá (UNIARAXÁ). \\ ${ }^{2}$ Professora Mestre do Centro Universitário do Planalto de Araxá (UNIARAXÁ). \\ ${ }^{3}$ Professora Doutora do Centro Universitário do Planalto de Araxá (UNIARAXÁ).
}

Cómo citar este artículo en edición digital: Silva, A.D.S., Ríos, O.F.L., \& Botelho, A.C.F. (2017). Presencia de estrés y otros factores de riesgo de enfermedad cardiovascular entre las enfermeras y abogados de una ciudad de Minas Gerais - Brasil.

Cultura de los Cuidados (Edición digital), 21(49). Recuperado de http://dx.doi.org/10.14198/cuid.2017.49.19

CCorrespondencia: Profa. Dra. Aline do Carmo França Botelho.Rua Antônio Barreto, 555, Fertiza, Araxá-MG. CEP: 38184-240. Telefone: 34-36625206. Correo electrónico: alinecfb@terra.com.br

Recibido: 18/03/2017; Aceptado: 15/05/2017

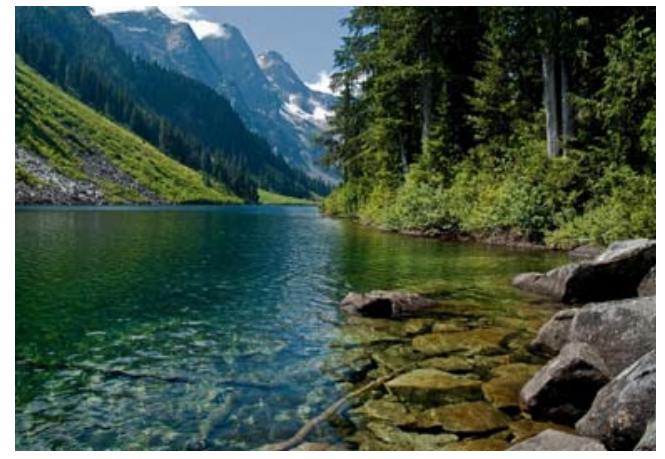

\section{ABSTRACT}

Cardiovascular disease (CVD) is an important cause of morbidity and mortality worldwide. This study aimed to evaluate stress and other risk factors for CVD in nurses and lawyers of a municipality of Minas Gerais (Brazil). The sample consisted of 20 nurses from the hospital area and 26 independent lawyers. Each volunteer answered two questionnaires, one on risk factors for CVD in general and the Inventory of Stress Symptoms for Adults
(ISSL). After it was found the body mass index (BMI), abdominal circumference (AC) and blood pressure of each volunteer. The results indicate that $55 \%$ of nurses and $30.9 \%$ of the lawyers present stress. The exhaustion stage is of particular concern, being found, respectively, $10 \%$ and $11.6 \%$ of nurses and lawyers. The findings relating to overweight / obesity, waist circumference of risk, sedentary lifestyle and intake of alcohol are worrying because increase the risk of CVD in the sample. It was concluded that there are risk factors for CVD and interventions are needed to improve health and quality of life of these professional.

Keywords: stress, cardiovascular diseases, nurses, lawyers.

\section{RESUMEN}

Las enfermedades cardiovasculares (ECV) son una causa importante de morbilidad y mortalidad en todo el mundo. Este estudio tuvo como objetivo evaluar el estrés y otros 
factores de riesgo de las enfermedades cardiovasculares en las enfermeras y abogados de un municipio de Minas Gerais (Brasil). La muestra estuvo conformada por 20 enfermeras de la zona hospitalaria y 26 abogados independientes. Cada voluntario respondió dos cuestionarios, uno sobre factores de riesgo de las enfermedades cardiovasculares en general y el Inventario de Síntomas de Estrés para Adultos (ISSL). Después se descubrió que el índice de masa corporal (IMC), la circunferencia abdominal (CA) y la presión sanguínea de cada voluntario. Los resultados indican que el 55\% de las enfermeras y el 30,9\% de los abogados presentes estrés. La etapa de agotamiento es especialmente preocupante, de ser encontrado, respectivamente, $10 \%$ y $11,6 \%$ de las enfermeras y abogados. Los hallazgos relacionados con el sobrepeso / obesidad, circunferencia de la cintura de riesgo, el sedentarismo y la ingesta de alcohol son preocupantes porque aumenta el riesgo de enfermedades cardiovasculares en la muestra. Se concluyó que existen factores de riesgo de ECV y se necesitan intervenciones para mejorar la salud y calidad de vida de estos profesionales.

Palabras clave: Estrés, enfermedades cardiovasculares, enfermeras, abogados.

\section{RESUMO}

As doenças cardiovasculares (DCV) constituem causa importante de morbidade e mortalidade em todo o mundo. Esse estudo objetivou avaliar o estresse e outros fatores de risco para DCV em enfermeiros e advogados de um munícipio de Minas Gerais (Brasil). A amostra foi composta por 20 enfermeiros da área hospitalar e com 26 advogados autônomos. Cada voluntário respondeu a dois questionários, um sobre fatores de risco para DCV em geral e o Inventário de Sintomas de Stress para Adultos
(ISSL). Após foi verificado o Índice de Massa Corporal (IMC), a Circunferência Abdominal (CA) e a pressão arterial de cada voluntário. Os resultados encontrados indicam que $55 \%$ dos enfermeiros e $30,9 \%$ dos advogados apresentam estresse. A fase de exaustão é especialmente preocupante, sendo encontrada, respectivamente, em $10 \%$ e $11,6 \%$ dos enfermeiros e advogados. Os achados referentes ao sobrepeso/obesidade, circunferência abdominal de risco, sedentarismo e ingestão de bebidas alcóolicas são preocupantes, pois elevam os riscos de DCV na amostra. É possível concluir que há fatores de risco para DCV e que intervenções são necessárias em prol da saúde e qualidade de vida desses profissionais.

Palavras-chave: estresse, doenças cardiovasculares, enfermeiros, advogados.

\section{INTRODUÇÃO}

As doenças crônicas não transmissíveis constituem causa importante de morbidade e mortalidade e são consideradas um dos problemas de saúde de maior magnitude no Brasil, dentre elas destacam-se as doenças cardiovasculares (DCV) como causa de 35\% dos óbitos em 2008 (Brasil, 2010).

As DCV mais importantes em termos de saúde coletiva são as doenças hipertensivas, as isquêmicas do coração e cerebrovasculares. Essas enfermidades têm em comum fatores de risco modificáveis, como tabagismo, uso abusivo de bebidas alcoólicas, sedentarismo, hábitos alimentares não saudáveis, excesso de peso e outros. Fatores não modificáveis também influenciam a ocorrência dessas doenças, como idade e herança genética. As DCV têm forte impacto na qualidade de vida dos indivíduos afetados, causam morte e incapacitação prematuras e por isso têm influência negativa 
sobre a economia, os sistemas de saúde e seguridade social (Malta et al., 2006; Silva, Luiz; Pereira, 2015).

Os profissionais da área da saúde são frequentemente submetidos a longas jornadas de trabalho, com 12 ou 24 horas de plantão, plantões noturnos, escassez de profissionais, baixas condições de trabalho, falta de recursos materiais, e, ainda precisam lidar com o estresse e o desgaste físico. São comuns problemas como distúrbios do sono, ansiedade, obesidade, hábitos de vida pouco saudáveis, consumo exagerado de bebidas alcoólicas e tabaco (Magalhães et al., 2014).

Somando-se a todos esses fatores encontra-se ainda a pressão no trabalho, a pequena ou quase nenhuma participação em tomada de decisões, falta de autonomia e o fato de lidar diretamente com pessoas, o que gera grande responsabilidade e, consequentemente, aumenta o nível de estresse (Ruviaro e Bardagi, 2010).

Em categorias profissionais na área de ciências humanas, destacam-se a classe dos advogados, estes lidam com vários fatores estressores e que levam ao risco aumentado de DCV. Suas atividades diárias envolvem grande pressão por prazos e decisões. Acrescente-se ainda a complexidade das leis e procedimentos legais, nível de exigência dos clientes, competição com outros profissionais, longas jornadas de trabalho, distanciamento da família por causa do trabalho, contato constante com conflitos, agressões, acusações, além de dispor pouco tempo para a prática de atividades físicas, manter hábitos alimentares adequados e lazer (Costa e Ferreira, 2014).

O estresse é uma reação interna do organismo para enfrentar situações percebidas como ameaçadoras. $\mathrm{O}$ estresse desenvolve-se em três fases: alerta, resistência e exaustão. A fase de alerta é aquela em que a pessoa precisa empenhar mais esforço e energia para executar o que está sendo exigido e enfrentar a situação desafiadora. Na segunda fase há um aumento na capacidade de resistência, que gera a busca pelo reequilíbrio, com a utilização de grande quantidade de energia e que pode desgastar a pessoa sem causa aparente, além de dificuldades na memória. Na última fase, a de exaustão, há uma quebra total da resistência; o indivíduo apresenta sintomas semelhantes à fase de alerta, porém com maior magnitude, produzindo grande exaustão psicológica em forma de depressão exaustão física (Silva e Martinez, 2005).

A grande maioria dos estudos sobre o estresse e outros fatores de risco para DCV contempla apenas profissões da área da saúde, da educação e estudantes universitários, estudos comparativos entre classes profissionais são raros na literatura científica. Diante disso, e, considerando a magnitude das DCV no Brasil e no mundo, a pesquisa tornou-se relevante. $\mathrm{O}$ objetivo do estudo foi avaliar o estresse e outros fatores de risco para doenças cardiovasculares em enfermeiros e advogados atuantes em Araxá-MG (Brasil).

\section{METODOLOGIA}

Trata-se de uma pesquisa de campo, exploratória, transversal e de natureza quantitativa realizada na cidade de Araxá, MG, Brasil, entre maio e setembro de 2015. A amostra foi composta por 20 enfermeiros da área hospitalar e com 26 advogados autônomos. Todos os participantes tinham mais de um ano de experiência na profissão.

Cada voluntário respondeu a dois questionários, um foi adaptado do Vigitel (Brasil, 2011) sobre fatores de risco para DCV e o Inventário de Sintomas de Stress para Adultos ISSL (Lipp, 2000). 
Para a verificação do IMC (índice de massa corporal) foi feita a pesagem e medição da altura. $\mathrm{O}$ peso foi aferido em balança digital da marca Relax Medic com capacidade máxima de $150 \mathrm{Kg}$ e divisão de $100 \mathrm{~g}$. Os voluntários foram posicionados no centro da balança, com o mínimo de roupas, eretos, com os braços estendidos ao longo do corpo e os pés juntos, conforme recomendação da cartilha de orientações para a coleta de dados antropométricos em serviços de saúde (Brasil, 2011). A estatura foi aferida com estadiômetro da marca Wiso. O IMC foi calculado com as medidas de peso e altura, de acordo com a seguinte fórmula: IMC $=$ peso $(\mathrm{kg}) / \operatorname{altura}^{2}(\mathrm{~m})$. Os pontos de corte de IMC adotados foram os preconizados: baixo peso (IMC < 18,5); eutrofia (IMC 18,524,99); sobrepeso (IMC 25-29,99) e obesidade (IMC $\geq 30,00$ ).

Para medida da circunferência abdominal (CA) foi utilizada uma fita métrica para medidas antropométricas. Ela pode ser obtida na menor curvatura localizada entre as costelas e a crista ilíaca com fita métrica flexível e inelástica sem comprimir os tecidos. Os pontos de corte adotados foram: risco aumentado para doenças cardiovasculares para mulheres $(\mathrm{CA}>80 \mathrm{~cm})$ e para homens $(\mathrm{CA}>94 \mathrm{~cm})$, e risco muito aumentado para mulheres $(\mathrm{CA}>$ $88 \mathrm{~cm}$ ) e para homens $(\mathrm{CA}>102 \mathrm{~cm})$ (Brasil, 2010).

Após foi realizada a aferição da pressão arterial dos entrevistados, sendo utilizado estetoscópio e esfigmomanômetro aneróide da marca Premium, para facilitar a coleta dos dados foi padronizado o braço esquerdo para a aferição. Foram adotados os seguintes pontos de corte segundo a VI Diretrizes Brasileiras de Hipertensão (SBC, 2010): ótima $<120 \mathrm{mmHg}$ $<80 \mathrm{mmHg}$, normal $<130 \mathrm{mmHg}<85 \mathrm{mmHg}$, limítrofe $\quad 130-139 \mathrm{mmHg} \quad-\quad 85-89 \mathrm{mmHg}$, hipertensão estágio I $140-159 \mathrm{mmHg} \quad$ 90-99mmHg, hipertensão estágio II 160$179 \mathrm{mmHg}-100-109 \mathrm{mmHg}$, hipertensão estágio III $\geq 180 \mathrm{mmHg} \geq 110 \mathrm{mmHg}$, hipertensão sistólica isolada $\geq 140 \mathrm{mmHg}<90 \mathrm{mmHg}$.

O projeto foi aprovado pelo Comitê de Ética em Pesquisa do Centro Universitário do Planalto de Araxá - UNIARAXÁ, protocolo de número 00445/08, e obedeceu as Diretrizes e Normas Regulamentadoras de Pesquisas Envolvendo Seres Humanos do Conselho Nacional de Saúde (Resolução 466/2012), que incorpora em seu contexto os quatro referenciais da bioética: autonomia, não maleficência, beneficência e justiça.

Os resultados foram tabulados e analisados através de estatística descritiva, para comparações utilizou-se o Teste Exato de Ficher ou o Teste Qui-quadrado, com nível de significância de $5 \%(p<0,05)$. Os softwares utilizados para a análise foram Excel e GraphPad InStat (3.0 for Windows).

\section{RESULTADOS}

A amostra envolveu uma participação majoritária feminina quando se avaliou os enfermeiros, sendo $90 \%$ do gênero feminino, já quanto aos advogados houve uma maior distribuição dos entrevistados, com predomínio do gênero masculino 53,84\%. As faixas etárias dos grupos entrevistados variaram de 23 a 62 anos, com média de idade de 35,80 anos.

Os resultados encontrados demonstraram que 55\% dos enfermeiros e 30,9\% dos advogados apresentam estresse. Dentre os enfermeiros $45 \%$ e $10 \%$ estão em fase de resistência e de exaustão, respectivamente. Quanto aos advogados, 19,3\% estão em fase de resistência e $11,6 \%$ estão em fase de exaustão. Não houve voluntários em fase de alerta e nem em fase de quase exaustão (Figura 1). 


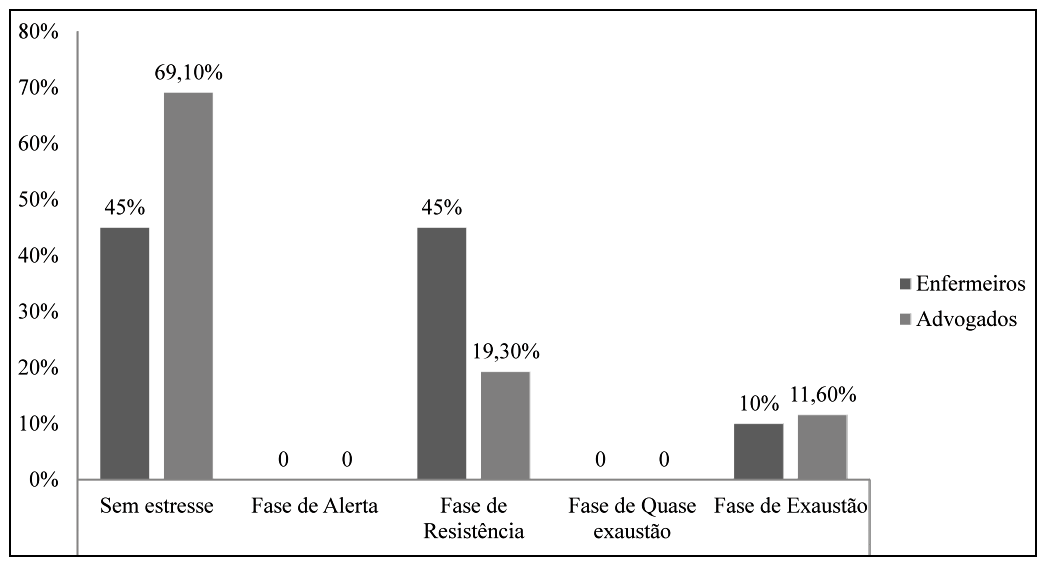

Figura 1 - Distribui $\square$ o dos entrevistados quanto ^ presen $\square$ a e fase do estresse.

De acordo com a figura 2, dentre os voluntários com estresse, houve uma predominância de sintomas psicológicos nas duas classes profissionais, com $82 \%$ e $78 \%$ para enfermeiros e advogados, respectivamente.

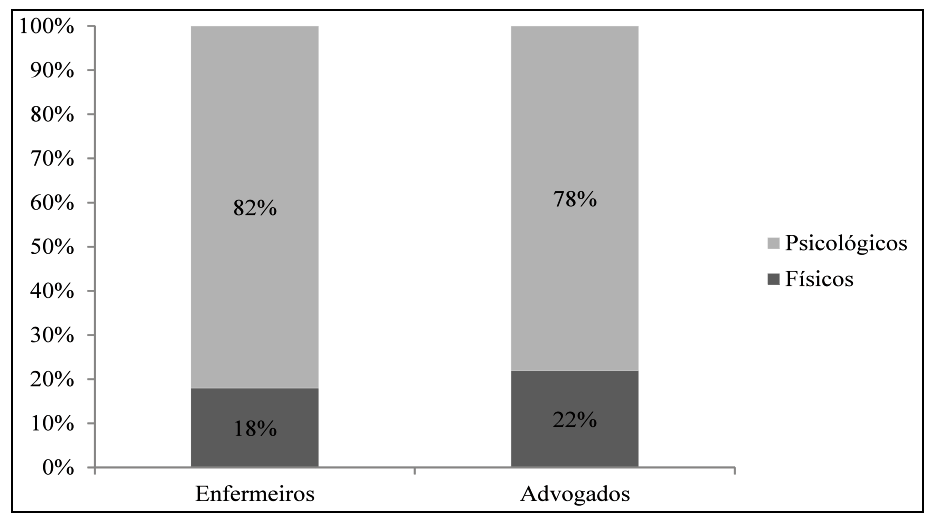

Figura 2 - Distribui $\square$ o dos entrevistados que apresentam estresse quanto ${ }^{\wedge}$ predomin $\square$ ncia de sintomas psicol $\sqsubset$ gicos e sintomas flsicos.

Observou-se que 30\% dos enfermeiros e 46,14\% dos advogados estão com sobrepeso/obesidade. Quanto à medida da circunferência abdominal apenas 25\% dos enfermeiros e 46,16\% dos advogados estão com a medida normal, a maioria está com risco aumentado ou muito aumentado (Tabela 1).

De acordo com a tabela 1 a amostra tem baixa prevalência de hipertensão e tabagismo. Entretanto, quanto ao sedentarismo e ingestão de bebidas alcóolicas os índices foram expressivos. Para o sedentarismo, os índices respectivos para enfermeiros e advogados foram 55\% e 34,56\%. E quanto ao consumo de bebidas alcóolicas 75\% dos enfermeiros e 73\% dos advogados afirmaram o uso frequente. 
Tabela 1 - Distribui $\square$ b dos entrevistados quanto ao ndice

de massa corporal (IMC), circunfer $\square$ ncia abdominal (CA), press $\square$ arterial, tabagismo, sedentarismo e ingest $\square$ de bebidas alc $\sqsubset$ olicas.

\begin{tabular}{|c|c|c|c|}
\hline & Classifica $\square 0$ & Enfermeiros & Advogados \\
\hline \multirow{5}{*}{ IMC } & Abaixo do peso & $1(5 \%)$ & $0(0 \%)$ \\
\hline & Peso normal & $13(65 \%)$ & $14(53,84 \%)$ \\
\hline & Acima do peso & $4(20 \%)$ & $9(34,61 \%)$ \\
\hline & Obesidade & $2(10 \%)$ & $3(11,53 \%)$ \\
\hline & Obesidade $\mathrm{m} \square$ rbida & $0(0 \%)$ & $0(0 \%)$ \\
\hline \multirow{3}{*}{$\mathbf{C A}$} & Normal & $5(25 \%)$ & $12(46,16 \%)$ \\
\hline & Risco aumentado & $9(45 \%)$ & $7(26,92 \%)$ \\
\hline & Risco muito aumentado & $6(30 \%)$ & $7(26,92 \%)$ \\
\hline \multirow{7}{*}{ Press $\lceil$ arterial } & $\square$ tima & $16(80 \%)$ & $22(84,61 \%)$ \\
\hline & Normal & $19(95 \%)$ & $24(92,30 \%)$ \\
\hline & Lim trofe & $3(15 \%)$ & $2(7,69 \%)$ \\
\hline & Hipertens $\sqsubset$ o est $\lceil$ gio 1 & $1(5 \%)$ & $2(7,69 \%)$ \\
\hline & Hipertens $\sqsubset$ o est $\ulcorner$ gio 2 & $0(0 \%)$ & $0(0 \%)$ \\
\hline & Hipertens $\square$ o est $\square$ gio 3 & $0(0 \%)$ & $0(0 \%)$ \\
\hline & Hipertens $\sqsubset 0$ arterial isolada & $1(5 \%)$ & $2(7,69 \%)$ \\
\hline \multirow{2}{*}{ Tabagismo } & Sim & $2(10 \%)$ & $5(19,24 \%)$ \\
\hline & $\mathrm{N} \sqsubset$ & $18(90 \%)$ & $21(80,76 \%)$ \\
\hline \multirow{2}{*}{ Sedentarismo } & Sim & $11(55 \%)$ & $9(34,56 \%)$ \\
\hline & $\mathrm{N} \sqsubset \mathrm{o}$ & $9(45 \%)$ & $17(65,28 \%)$ \\
\hline Uso frequente de & Sim & $15(75 \%)$ & $19(73,07 \%)$ \\
\hline bebidas alc olicas & $\mathrm{N} \sqsubset \mathrm{o}$ & $5(25 \%)$ & $7(26,93 \%)$ \\
\hline
\end{tabular}

\section{DISCUSSÃO}

Há vários fatores de risco para as DCVs, alguns são considerados não modificáveis, como idade, genética e etnia, mas a maioria deles está relacionada ao estilo de vida, sendo passíveis de prevenção. Dentre os fatores modificáveis está o estresse, que é um estado que altera o bem-estar do indivíduo, reduz a qualidade de vida e traz prejuízos para a saúde.

A associação entre o estresse e DCV não é novidade na ciência, mas os mecanismos envolvidos ainda não são totalmente conhecidos. Várias possibilidades têm sido propostas, incluindo hiperatividade do sistema nervoso autônomo e alterações hormonais, que vão convergir para disfunção endotelial e indesejáveis consequências cardiovasculares, com alterações cardíacas e hematológicas. É provável que a ativação da cascata inflamatória, associada ao desequilíbrio oxidativo, represente o principal componente fisiopatológico que faz do estresse um fator 
de risco para DCV (Golbidi, Frisbee e Laher, 2015).

Neste estudo a presença de estresse na amostra foi um achado muito relevante, inclusive com alguns voluntários em fase de exaustão, que é a mais grave, é o momento em que o organismo se vê exaurido e fica suscetível as doenças (Lipp, 2001). Segundo Pafaro e Martino (2004) a exaustão é a quebra do equilíbrio do organismo e está associada a hipertensão arterial, depressão, ansiedade, problemas sexuais, doenças dermatológicas, infarto e até morte súbita.

No estudo de Costa, Lima e Almeida (2003) na cidade de Fortaleza, Ceará (Brasil), com 42 enfermeiros, 38\% dos voluntários apresentavam estresse e $7,1 \%$ dos enfermeiros estavam em fase de exaustão, esses percentuais são menores que os obtidos no presente estudo, que foram $55 \%$ e $10 \%$, respectivamente.

No estudo de Loricchio e Leite (2012), com Bacharéis em Direito em São Paulo (SP - Brasil), foi demonstrado que grande parte dos bacharéis (71\%) estava em alguma fase de estresse, índice mais alto que o obtido aqui. Esse índice alto possivelmente deve-se ao fato de que a amostra de São Paulo foi composta por profissionais em fase de preparação para ingresso na Ordem dos Advogados do Brasil, momento de grande tensão para a classe.

Neste estudo tanto enfermeiros quanto advogados tiveram predominantemente sintomas psicológicos, corroborando com Camelo e Angerami (2004), em estudo com trabalhadores de cinco núcleos de saúde da família de Ribeirão Preto (SP - Brasil), eles também encontraram a predominância de sintomas psicológicos. Já no estudo de Carvalho e Malagris (2007), com 31 profissionais da área da saúde, de nível superior, atuantes em um Posto de Assistência Médica (PAM) do Rio de Janeiro
(RJ - Brasil), houve predomínio de sintomas físicos.

Além do estresse, outros fatores de risco modificáveis são também muito relevantes para as DCV. Os baixos índices encontrados quanto à hipertensão e tabagismo são aspectos muito positivos. Nos últimos anos têm sido frequentes campanhas antitabagistas, o que pode ter contribuído para a baixa frequência deste vício na amostra. Entretanto, os achados referentes ao sobrepeso/obesidade, circunferência abdominal de risco, sedentarismo e ingestão de bebidas alcóolicas são preocupantes, pois elevam os riscos de DCV na amostra.

Vale ainda salientar que não foram encontradas diferenças estatisticamente significativas entre enfermeiros e advogados para os parâmetros analisados, o que indica que, mesmo exercendo funções muito distintas, profissionais de diferentes áreas podem ter similaridades quanto ao estilo de vida e risco para DCV.

\section{CONCLUSÃO E CONSIDERAÇÕES FI- NAIS}

Diante dos resultados obtidos é possível concluir que tanto os enfermeiros quanto os advogados apresentam fatores de risco para o desenvolvimento de doenças cardiovasculares. A prevalência de estresse, especialmente a fase de exaustão, encontrada em alguns voluntários é de grande relevância no contexto saúde-doença. Além disso, o excesso de gordura corporal, o sedentarismo e a ingestão frequente de bebidas alcóolicas elevam o risco cardiovascular da amostra.

Este foi um dos poucos estudos que buscou comparar os fatores de risco entre profissionais de áreas diferentes e a evidenciar semelhanças. A presença de muitos agentes estressores, sobrecarga de trabalho e pouco tempo para o cultivo de hábitos de vida saudáveis, podem 
ser pontos de aproximação entre enfermeiros e advogados.

A expectativa de que outras profissões também possuem risco cardiovascular aumentado, instiga pesquisas futuras e também alerta para a necessidade de ações em prol da saúde nos diferentes ambientes de trabalho e para várias classes profissionais.

\section{REFERÊNCIAS}

- Brasil. Ministério da Saúde. (2010). Diretrizes Brasileiras de Obesidade. 3 ed. São Paulo: Abeso.

- Brasil. Ministério da saúde. (2011). Plano de ações estratégicas para o enfrentamento das doenças crônicas não transmissíveis no Brasil 2011-2022. Disponível em htpp://bvsms.saude.gov.br/bvs/publicacoes/plano_ acoes_enfrent_dcnt_2011.pdf.

- Brasil. Ministério da Saúde. Secretaria de Vigilância em Saúde. Departamento de Análise de Situação de Saúde. (2010). Saúde Brasil 2009: uma análise da situação de saúde e da agenda nacional e internacional de prioridades em saúde. Brasília: Ministério da Saúde.

- Camelo, S.H., \& Angerami, E.L.S. (2004). Sintomas de estresse nos trabalhadores atuantes em cinco núcleos de saúde da família. Rev. Latino-Am. Enfermagem. 12(1), 14-21.

- Costa, J.R.A., Lima, J.V., \& Almeida, P.C. (2003). Stress no trabalho do enfermeiro. Rev Esc Enfermagem USP. 37(3), 63-71.

- Costa, M.F.A.A., \& Ferreira, M.C. (2014). Fontes e reações de estresse em advogados brasileiros. Paidéia. 24(57), 49-56.

- Golbidi, S., Frisbee, J.C., \& Laher, I. (2015). Crônicas de estresse afeta a cardiovascular sistema: modelos animais e os resultados clínicos. Am J Physiol coração Circ Physiol. 308(12), H1476-98.

- Carvalho, L., \& Malagris, L.E.N. (2007). Avaliação do nível de stress em profissionais de saúde. Estud. pesqui. psicol. 7(3), 570-582.

- Lipp, M.E.N. (2000). Manual do Inventário de Sintomas de Stress para Adultos de Lipp (ISSL). São Paulo: Casa do Psicólogo.

- Lipp, M.E.N. O stress emocional e seu tratamento. (2001). In: Rangé, B. Psicoterapias Cognitivo-comportamentais: um diálogo com a psiquiatria. Porto Alegre: Artmed.

- Loricchio, T.M.B., \& Leite, J.R. (2012). Estresse, ansiedade, crenças de autoeficácia e o desempenho dos bacharéis em Direito. Aval. psicol. 11(1), 37-47.

- Magalhães, F.J., Mendonça, L.B.A., Rebouças, C.B.A., Lima, F.E.T., Custódio, I.L., \& Oliveira, S.C. (2014). Fatores de risco para doenças cardiovasculares em profissionais de enfermagem: estratégias de promoção da saúde. Rev. bras. enferm. 67(3), 394-400.
- Malta, D.C., Cezário, A.C., Moura, L., Morais Neto, O.L., \& Silva Júnior J.B. (2006). A construção da vigilância e prevenção das doenças crônicas não transmissíveis no contexto do Sistema Único de Saúde. Epidemiol Serv Saúde. 15(3), 47-64.

- Pafaro, R.C., \& De Martino, M.M.F. (2004). Estudo do estresse do enfermeiro com dupla jornada de trabalho em um hospital de oncologia pediátrica de Campinas. Rev. esc. enferm. USP. 38(2), 152-160.

- Ruviaro, M.F.S., \& Bardagi, M.P. (2010). Síndrome de Burnout e satisfação no trabalho em profissionais da área de enfermagem do interior do RS. Barbarói. 33(2),194-216.

- SBC. Sociedade Brasileira de Cardiologia. VI Diretrizes Brasileiras de Hipertensão. (2010). Disponível em http:// publicacoes.cardiol.br/consenso/2010/Diretriz_hipertensao_associados.pdf.

- Silva, E.A.T., \& Martinez, A. (2005). Diferença em nível de stress em duas amostras: capital e interior do estado de São Paulo. Estud. psicol. (Campinas). 22(1): 53-61.

- Silva, S.M., Luiz, R.R., \& Pereira, R.A. (2015). Fatores de risco e proteção para doenças cardiovasculares em adultos de Cuiabá, Mato Grosso, Brasil. Rev. bras. epidemiol. 18(2), 425-438. 